\title{
Investigating the nature of the Fried Egg nebula ${ }^{\star}$ CO mm-line and optical spectroscopy of IRAS 17163-3907
}

\author{
S. H. J. Wallström ${ }^{1}$, S. Muller ${ }^{1}$, E. Lagadec ${ }^{2}$, J. H. Black ${ }^{1}$, R. D. Oudmaijer ${ }^{3}$, K. Justtanont ${ }^{1}$, \\ H. van Winckel ${ }^{4}$, and A. A. Zijlstra ${ }^{5}$ \\ ${ }^{1}$ Department of Earth and Space Sciences, Chalmers University of Technology, Onsala Space Observatory, 439-92 Onsala, Sweden \\ e-mail: sofia.wallstrom@chalmers.se \\ 2 Department of Astronomy, Cornell University, Ithaca, NY 14853-6801, USA \\ 3 School of Physics and Astronomy, University of Leeds, Leeds LS2 9JT, UK \\ 4 Instituut voor Sterrenkunde, K.U. Leuven, Celestijnenlaan, 200D, 3001 Leuven, Belgium \\ 5 Jodrell Bank Center For Astrophysics, The University of Manchester, Manchester, M13 9PL, UK
}

Received 20 March 2013 / Accepted 12 December 2014

\section{ABSTRACT}

\begin{abstract}
Through CO mm-line and optical spectroscopy, we investigate the properties of the Fried Egg nebula IRAS 17163-3907, which has recently been proposed to be one of the rare members of the yellow hypergiant class. The CO $J=2-1$ and $J=3-2$ emission arises from a region within $20^{\prime \prime}$ of the star and is clearly associated with the circumstellar material. The CO lines show a multi-component asymmetrical profile, and an unexpected velocity gradient is resolved in the east-west direction, suggesting a bipolar outflow. This is in contrast with the apparent symmetry of the dust envelope as observed in the infrared. The optical spectrum of IRAS 17163-3907 between 5100 and $9000 \AA$ was compared with that of the archetypal yellow hypergiant IRC+10420 and was found to be very similar. These results build on previous evidence that IRAS 17163-3907 is a yellow hypergiant.
\end{abstract}

Key words. circumstellar matter - stars: AGB and post-AGB - stars: mass-loss - stars: individual: IRAS 17163-3907

\section{Introduction}

Yellow hypergiants (YHGs) are a class of massive evolved stars. The YHG phase is thought to be short lived, and examples of such stars are rare, with only about a dozen known in our galaxy (Oudmaijer et al. 2009). However, YHG stars are characterised by intense mass-loss and can greatly impact the chemical enrichment of the interstellar medium, both with their massive winds and with their ultimate explosions as core-collapse supernovae.

According to de Jager (1998), the defining characteristics of YHGs are $\mathrm{Ia}^{+}$luminosity class, based on visible spectra, with $\mathrm{H} \alpha$ emission in one or more broad components and absorption lines that are broader than those of Ia supergiants of a similar spectral type. The $\mathrm{H} \alpha$ emission is a signature of an extended atmosphere and mass loss, while the comparatively broad absorption lines signify large-scale photospheric motions such as pulsation.

These empirical characteristics have a physical interpretation, placing the YHGs in a blueward evolutionary loop on the Hertzsprung-Russell diagram after the red supergiant phase. Only stars with initial masses between 20 and $40 M_{\odot}$ can take this path, and the timescale of the YHG phase is of the order of 100-1000 years, accounting for the rarity of this type of star. A YHG may subsequently evolve through a phase as a luminous blue variable, and finally become a Wolf-Rayet star (Oudmaijer et al. 2009).

Among the most well-studied YHGs are HD 179821 (also known as IRAS 19114+0002 or AFGL 2343) and IRC+10420,

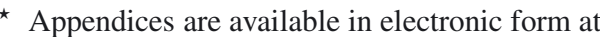
http://www . aanda.org
}

both of which have prominent infrared excesses and dusty circumstellar envelopes, the latter having suffered a major ejection within the last 600 years (Humphreys et al. 1997, 2002; Oudmaijer et al. 1996). Very few YHG stars show resolved envelopes (de Jager 1998), and studies of their mass-loss are limited (e.g. Castro-Carrizo et al. 2007; Dinh-V.-Trung et al. 2009).

This paper focuses on the source IRAS 17163-3907 (also known as Hen 3-1379, and hereafter referred to as IRAS 17163). It was discovered by Henize (1976) and was first classified as a post-AGB star by Le Bertre et al. (1989). Using interstellar K I absorption in the optical spectrum, Lagadec et al. (2011b) placed a lower limit of $3.6 \mathrm{kpc}$ on the distance to the star. An upper limit of $\sim 4.7 \mathrm{kpc}$ was derived from the maximum visual extinction implied by diffuse interstallar band absorptions. Consequently, IRAS 17163 is at a distance four times larger than the distance of $\sim 1 \mathrm{kpc}$ estimated by Le Bertre et al. (1989). The revised luminosity of $\sim 5 \times 10^{5} L_{\odot}$, along with its position on a temperature-luminosity diagram close to the brightest YHGs like IRC +10420 , means that IRAS 17163 is almost certainly a member of the yellow hypergiant class.

Despite being one of the brightest objects in the mid-IR sky, IRAS 17163 has so far been the subject of limited investigations. Mid-IR images of IRAS 17163 were obtained by Lagadec et al. (2011a,b) using the VLT-VISIR instrument. Two concentric spherical dusty shells were resolved within $2.5^{\prime \prime}$ of the central star, with a warm $(\sim 200 \mathrm{~K})$ dust mass of $0.04 M_{\odot}$. The presence of two shells suggests an episodic enhancement of the mass-loss rate on timescales of a few hundred years. IRAS 17163 was also imaged with Herschel (Hutsemékers et al. 2013), revealing a symmetric dust shell $\sim 25^{\prime \prime}$ in radius around 
the star. The mass of cool $(60 \mathrm{~K})$ dust in this shell is inferred to be as high as $\sim 0.17 M_{\odot}$, implying a huge (although poorly constrained because of the unknown gas/dust ratio) circumstellar gas mass. However, dust imaging lacks kinematic information, preventing us from studying the mass loss and its timescale in more detail. In this paper, we present the first observations of CO rotational lines around IRAS 17163 and an optical spectrum of the star between 5100 and $9000 \AA$.

\section{Observations}

\subsection{APEX observations}

IRAS 17163 was observed with the Atacama Pathfinder EXperiment (APEX) telescope ${ }^{1}$ on April 9-12, 2014. A $\sim 100^{\prime \prime} \times 100^{\prime \prime}$ on-the-fly map in the CO $J=2-1$ and $J=3-2$ transitions was performed, centred on the star at RA 17:19:49.33, Dec -39:10:37.9 (J2000). The data were taken with a scan spacing of $9^{\prime \prime}$ in $\mathrm{CO} 2-1$ (main beam size 27") and $6^{\prime \prime}$ in CO 3-2 (beam size 18").

The direction of IRAS 17163 lies close to the complex starforming regions RCW 121 and RCW 122 (see Fig. 1), which show bright $\mathrm{CO}$ emission at velocities between $v_{\mathrm{LSR}}=-40$ and $0 \mathrm{~km} \mathrm{~s}^{-1}$, extended over a region of several square arcminutes (Arnal et al. 2008). We adopted an on/off position-switching calibration scheme, carefully selecting an off-position free from CO emission, $\sim 20^{\prime}$ south-east of the source.

The data were reduced within the standard single-dish data reduction package CLASS ${ }^{2}$. The spectra have a total velocity coverage of 5720 and $3810 \mathrm{~km} \mathrm{~s}^{-1}$ in CO 2-1 and 3-2, respectively. There are no line detections in either spectrum outside the velocity interval -40 to $+110 \mathrm{~km} \mathrm{~s}^{-1}$.

The components between -40 and $0 \mathrm{~km} \mathrm{~s}^{-1}$ correspond to CO emission from the RCW 121 and RCW 122 complexes (Arnal et al. 2008). An additional emission feature is seen between $v_{\text {LSR }}=50-110 \mathrm{~km} \mathrm{~s}^{-1}$ (Fig. 2). In contrast to the widespread $\mathrm{CO}$ features in the velocity range -40 to $0 \mathrm{~km} \mathrm{~s}^{-1}$, this component is confined to within $\sim 20^{\prime \prime}$ of IRAS 17163 (Figs. 3, 4) and is clearly associated with the circumstellar envelope. The $\mathrm{CO}$ radial velocity, $\sim 70 \mathrm{~km} \mathrm{~s}^{-1}$, is also completely incompatible with galactic rotation at any distance towards IRAS 17163.

\subsection{Optical spectrum from Mercator}

IRAS 17163 was observed on August 10, 2009, with the fibrefed spectrograph HERMES (Raskin et al. 2011) attached to the 1.2-m Mercator telescope at Roque de los Muchachos observatory, Spain. The fibre has an aperture of $2.5^{\prime \prime}$ on the sky. Four exposures of $20 \mathrm{~min}$ each were taken in concatenation, and the data were reduced with the dedicated pipeline. The resulting flatfielded, merged, and wavelength-calibrated spectrum was normalized by the continuum level and is presented in Appendix B. Note that the spectrum was not corrected for telluric features. The spectral resolution is $\sim 4 \mathrm{~km} \mathrm{~s}^{-1}$ and the signal-to-noise ratio ranges between $\sim 5-100$ depending on the wavelength, with lower values in the blue due to the severe reddening of the star.

\footnotetext{
This publication is based on data acquired with the Atacama Pathfinder Experiment (APEX). APEX is a collaboration between the Max-Planck-Institut fur Radioastronomie, the European Southern Observatory, and the Onsala Space Observatory.

2 http://www.iram.fr/IRAMFR/GILDAS
}

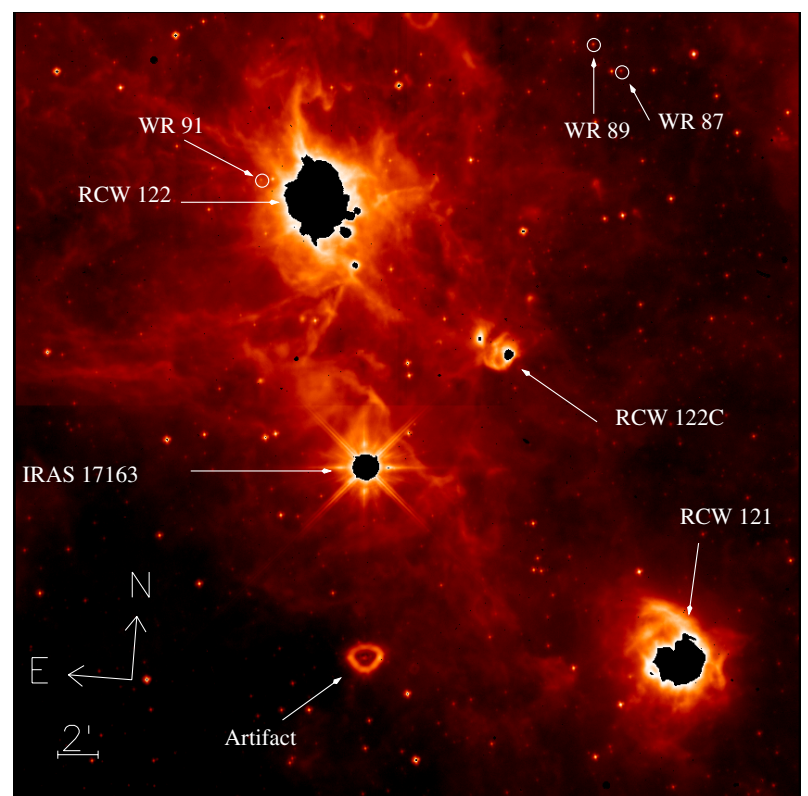

Fig. 1. WISE W3 band $(11.56 \mu \mathrm{m})$ image of the field around IRAS 17163, showing the nearby star-forming regions RCW 121 and 122 .

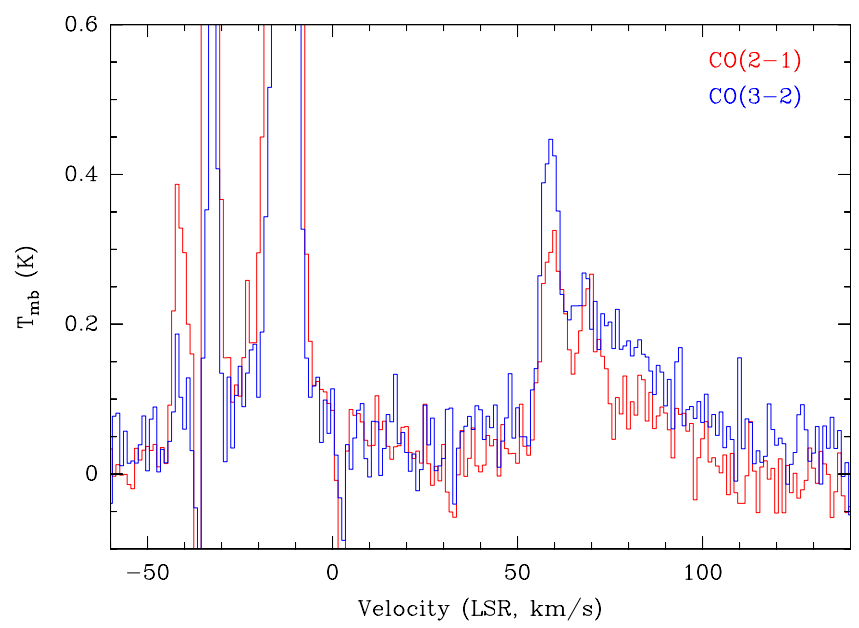

Fig. 2. APEX spectra (velocity resolution $1 \mathrm{~km} \mathrm{~s}^{-1}$ ) of the CO 2-1 (red) and 3-2 (blue) emission, averaged over the central 20" around IRAS 17163. The emission between -40 and $0 \mathrm{~km} \mathrm{~s}^{-1}$ is interstellar, while the emission between 50 and $110 \mathrm{~km} \mathrm{~s}^{-1}$ is associated with the star.

\section{Results}

\subsection{CO emission}

The CO $J=2-1$ and $J=3-2$ emission lines are broad $\left(\sim 60 \mathrm{~km} \mathrm{~s}^{-1}\right)$ and asymmetrical, with peaks between $v_{\mathrm{LSR}}=$ $50-70 \mathrm{~km} \mathrm{~s}^{-1}$ and a fainter plateau between $70-110 \mathrm{~km} \mathrm{~s}^{-1}$ (Fig. 2). Integrating over these blue $\left(50-70 \mathrm{~km} \mathrm{~s}^{-1}\right)$ and red $\left(70-110 \mathrm{~km} \mathrm{~s}^{-1}\right.$ ) emission components shows that the $\mathrm{CO}$ emission is concentrated within $\sim 20^{\prime \prime}$ of the star and that there is a clear spatial separation between the two components (Fig. 3). In CO 2-1 the blue and red components' emission peaks are offset from the star by $\sim 14^{\prime \prime}$ and $4^{\prime \prime}$, respectively, giving a spatial separation of $\sim 18^{\prime \prime}$, in a mainly east-west direction. This velocity gradient is resolved within the APEX beam and suggests an asymmetrical outflow with a velocity of $\sim 30 \mathrm{~km} \mathrm{~s}^{-1}$. The velocity gradient is less apparent in $\mathrm{CO} 3-2$, partly because the 
S. H. J. Wallström et al.: Investigating the nature of the Fried Egg nebula
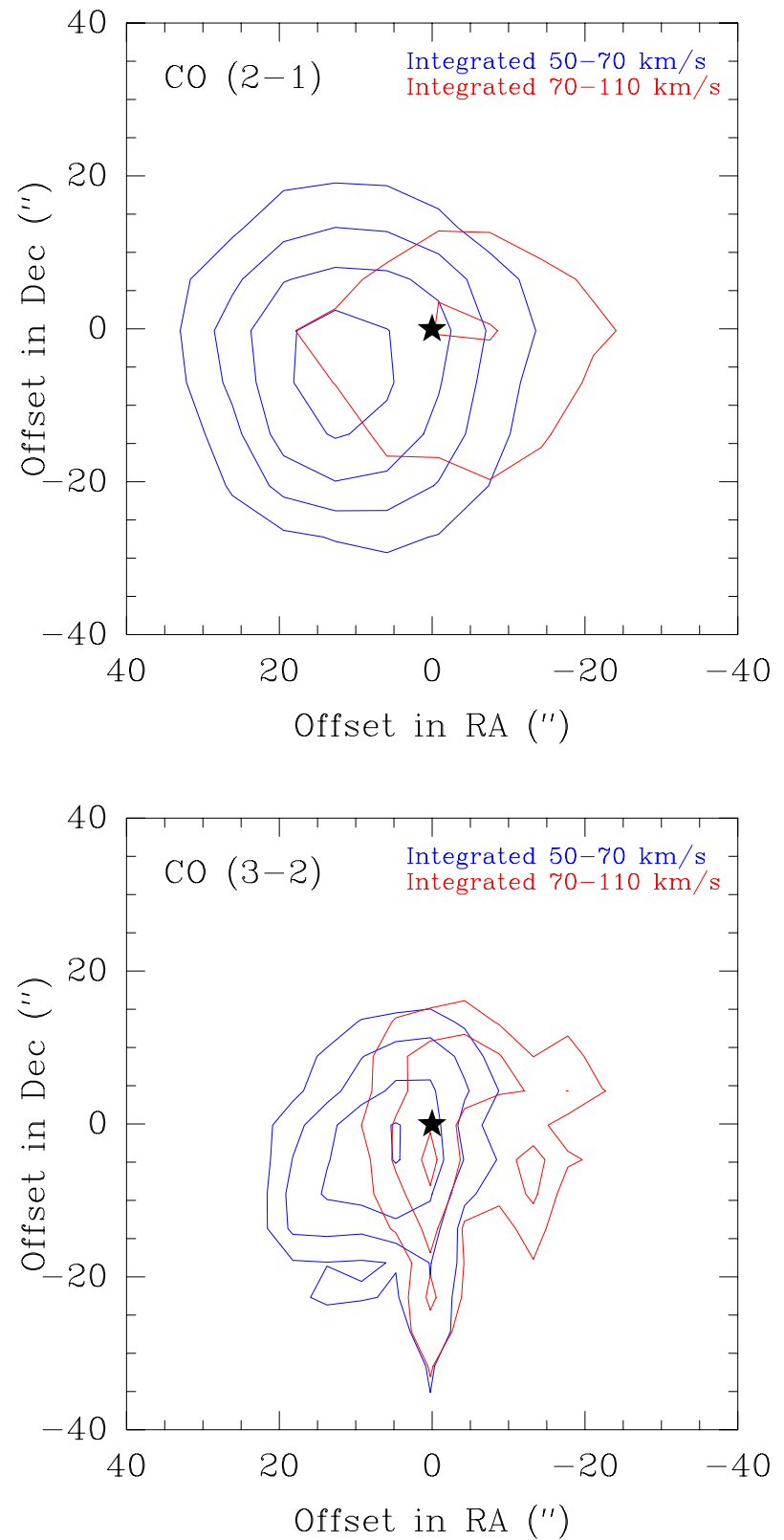

Fig. 3. Contours of the integrated $\mathrm{CO} 2-1$ and 3-2 emission between $50-70 \mathrm{~km} \mathrm{~s}^{-1}$ (blue) and $70-110 \mathrm{~km} \mathrm{~s}^{-1}$ (red). All contours start at $5 \sigma$ and are spaced by $5 \sigma\left(1 \sigma=0.27 \mathrm{~K} \mathrm{~km} \mathrm{~s}^{-1}\right.$ for CO 2-1 and $0.33 \mathrm{~K} \mathrm{~km} \mathrm{~s}^{-1}$ for $\mathrm{CO} 3-2$ ). The main beam sizes are $27^{\prime \prime}$ for $\mathrm{CO} 2-1$ and $18^{\prime \prime}$ for $\mathrm{CO} 3-2$. The star at $(0,0)$ marks the position of IRAS 17163.

red component has a low signal-to-noise ratio. In addition, the CO 3-2 emission is generally concentrated closer to the star, with the blue and red components' emission peaks offset from the star by $\sim 6^{\prime \prime}$ east and $5^{\prime \prime}$ south, respectively. This may be because the gas closer to the star is warmer and hence brighter in CO 3-2 than $2-1$. The $\mathrm{CO}(3-2) /(2-1)$ peak intensity ratio is $\sim 1.4$ at the star, and decreases to $\sim 1.2$ at $\left(+10^{\prime \prime}, 0\right)$.

Previous infrared observations have found a warm dust mass of $0.04 M_{\odot}$ in shells within $2.5^{\prime \prime}$ of the star (Lagadec et al. $2011 \mathrm{~b}$ ), and a cooler dust ring peaking $\sim 25^{\prime \prime}$ from the star (extending in radius from $18^{\prime \prime}$ to $40^{\prime \prime}$ ), with a dust mass of $0.17 M_{\odot}$ (Hutsemékers et al. 2013). This large amount of dust implies a significant gas mass, especially in the $25^{\prime \prime}$ ring, but the CO observations show little emission in this area (Fig. 4). The
$\mathrm{CO}$ emission is found mainly within $\sim 20^{\prime \prime}$ of the star, which is most likely due to the $\mathrm{CO}$ farther away being photodissociated by the interstellar UV radiation field. The asymmetry of the $\mathrm{CO}$ emission contrasts with the apparent symmetry of the dust rings at both $\sim 2.5^{\prime \prime}$ and $\sim 25^{\prime \prime}$ from the star. However, we note that a slight asymmetry can be seen in the Hutsemékers et al. (2013) dust image, along the same direction as the CO velocity gradient.

The asymmetry of the $\mathrm{CO}$ line profile and the velocity gradient mean we cannot model the mass loss as a spherically symmetric wind. This precludes us from reliably estimating the mass-loss rate. However, catalogues of AGB and post-AGB stars indicate that a $\mathrm{CO}$ envelope of $\sim 20^{\prime \prime}$ would require a large sustained mass-loss rate of around $10^{-5} M_{\odot} \mathrm{yr}^{-1}$ (Loup et al. 1993). This is in the range of expected mass-loss rates for YHGs.

To give a rough estimate of the circumstellar gas mass, we consider that if the integrated CO line intensity $\left(5.85 \mathrm{~K} \mathrm{~km} \mathrm{~s}^{-1}\right)$ were that of a gravitationally bound spherical interstellar cloud of $20^{\prime \prime}$ radius at a distance of $4 \mathrm{kpc}$, then the application of a standard $\mathrm{CO} / \mathrm{H}_{2}$ conversion factor of $2 \times 10^{20} \mathrm{~cm}^{-2} /\left(\mathrm{K} \mathrm{km} \mathrm{s}^{-1}\right)$ would imply a gas mass $\sim 6 M_{\odot}$. By coincidence, this is similar to the $\sim 7 M_{\odot}$ mass of the $25^{\prime \prime}$ dust ring derived by Hutsemékers et al. (2013), who assumed a gas-to-dust mass ratio of 40 .

\subsection{Optical spectroscopy}

The optical spectrum of IRAS 17163 is rich in lines, mainly in emission, but also some in absorption. Of the identified lines, the largest number correspond to Fe II, Cr II, and Ca II in emission and $\mathrm{N} \mathrm{I}$ and $\mathrm{H} \mathrm{I}$ in absorption. Interstellar absorption lines of $\mathrm{KI}$ and diffuse interstellar bands (DIBs) have velocities corresponding to the $\mathrm{CO}$ emission from the surrounding star-forming regions (see Fig. 5).

The brightest line, $\mathrm{H} \alpha$ (at $6563 \AA$ ), has a P-Cygni profile with a full width at half maximum of $\sim 140 \mathrm{~km} \mathrm{~s}^{-1}$ (see Fig. 6) and broad wings extending out to a width of $\sim 2400 \mathrm{~km} \mathrm{~s}^{-1}$. A P-Cygni line profile is indicative of a substantial outflow of material, and the broad $\mathrm{H} \alpha$ wings may be due to scattering by free electrons, as suggested by Humphreys et al. (2002) for IRC +10420 . Three Ca II lines (at 8498, 8542, and $8662 \AA$ ) also show P-Cygni profiles, with line widths similar to that of $\mathrm{H} \alpha$. A relatively large number $(\sim 50)$ of lines remains unidentified in the spectrum.

The central velocities of various lines range from +10 to $+43 \mathrm{~km} \mathrm{~s}^{-1}$ (see Fig. 6), but both $\mathrm{H} \alpha$ and the narrow ( $F W H M=$ $25 \mathrm{~km} \mathrm{~s}^{-1}$ ) Fe II lines are centred on a velocity of $+18 \mathrm{~km} \mathrm{~s}^{-1}$. Given their excitation, the Fe II lines most likely arise near the stellar photosphere and are indicative of the stellar velocity. However, their velocity is blueshifted by about $50 \mathrm{~km} \mathrm{~s}^{-1}$ relative to the velocity of the $\mathrm{CO}$ emission. Possible explanations for this are discussed in Sect. 4.1.

The absolute magnitude $\left(M_{V}\right)$ of IRAS 17163 can be estimated from the equivalent width $\left(W_{\lambda}\right)$ of the O I (7774) triplet, which has been shown to be a good indicator of $M_{V}$ for stars with spectral types A to $\mathrm{G}$ (Arellano Ferro et al. 2003, and references therein). We measure $W_{\lambda}(\mathrm{O} \mathrm{I}(7774))=3.14 \pm 0.01 \AA$, which is just outside the range of the $W_{\lambda}(\mathrm{OI})-M_{V}$ calibration by Arellano Ferro et al. (2003). Nevertheless, a straight extrapolation suggests an $M_{V}$ close to -10, comparable with the values similarly derived for the two YHGs IRC+10420 and HD 179821 (Oudmaijer et al. 2009). 

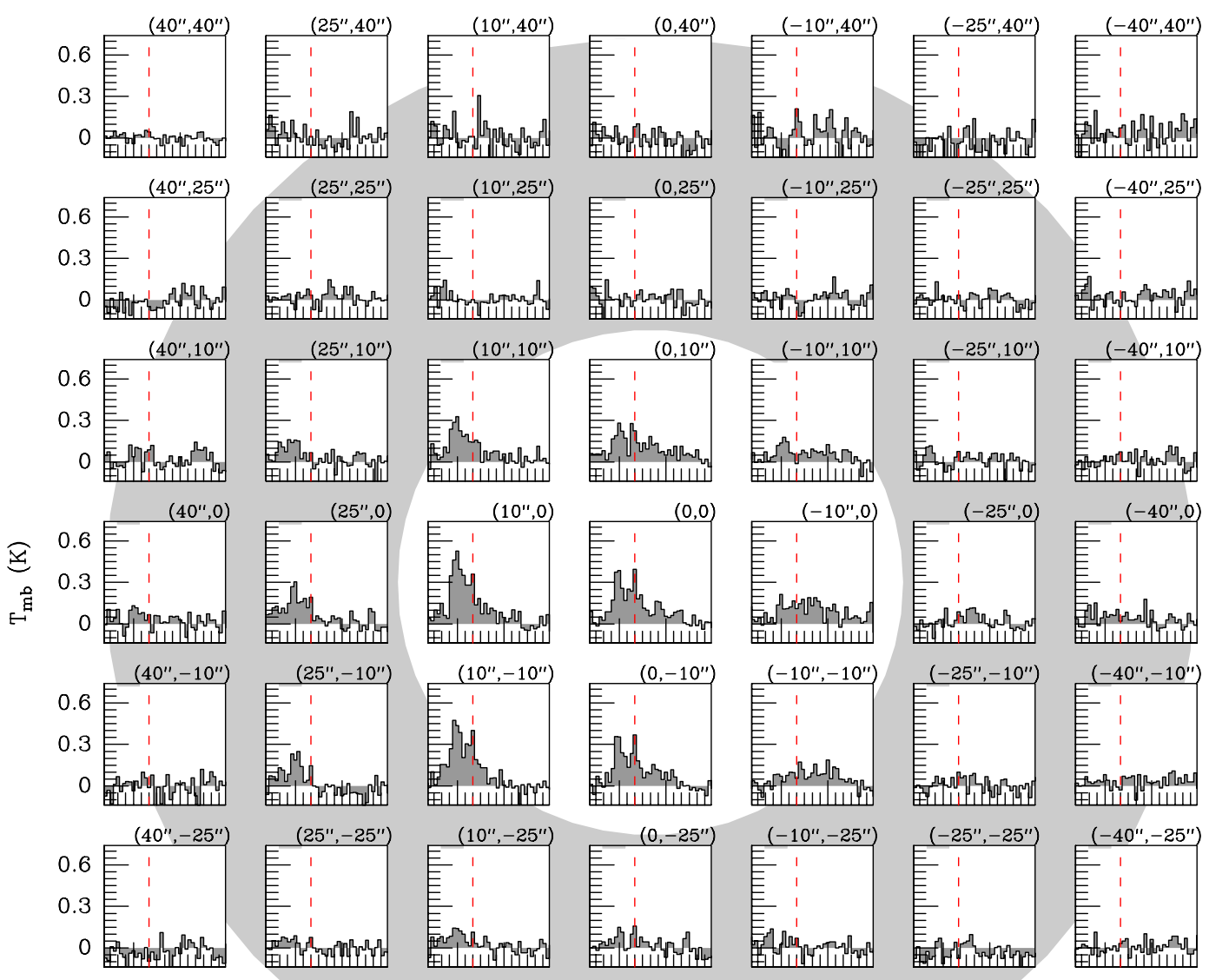

$\left(-10^{\prime \prime}, 10^{\prime \prime}\right)$
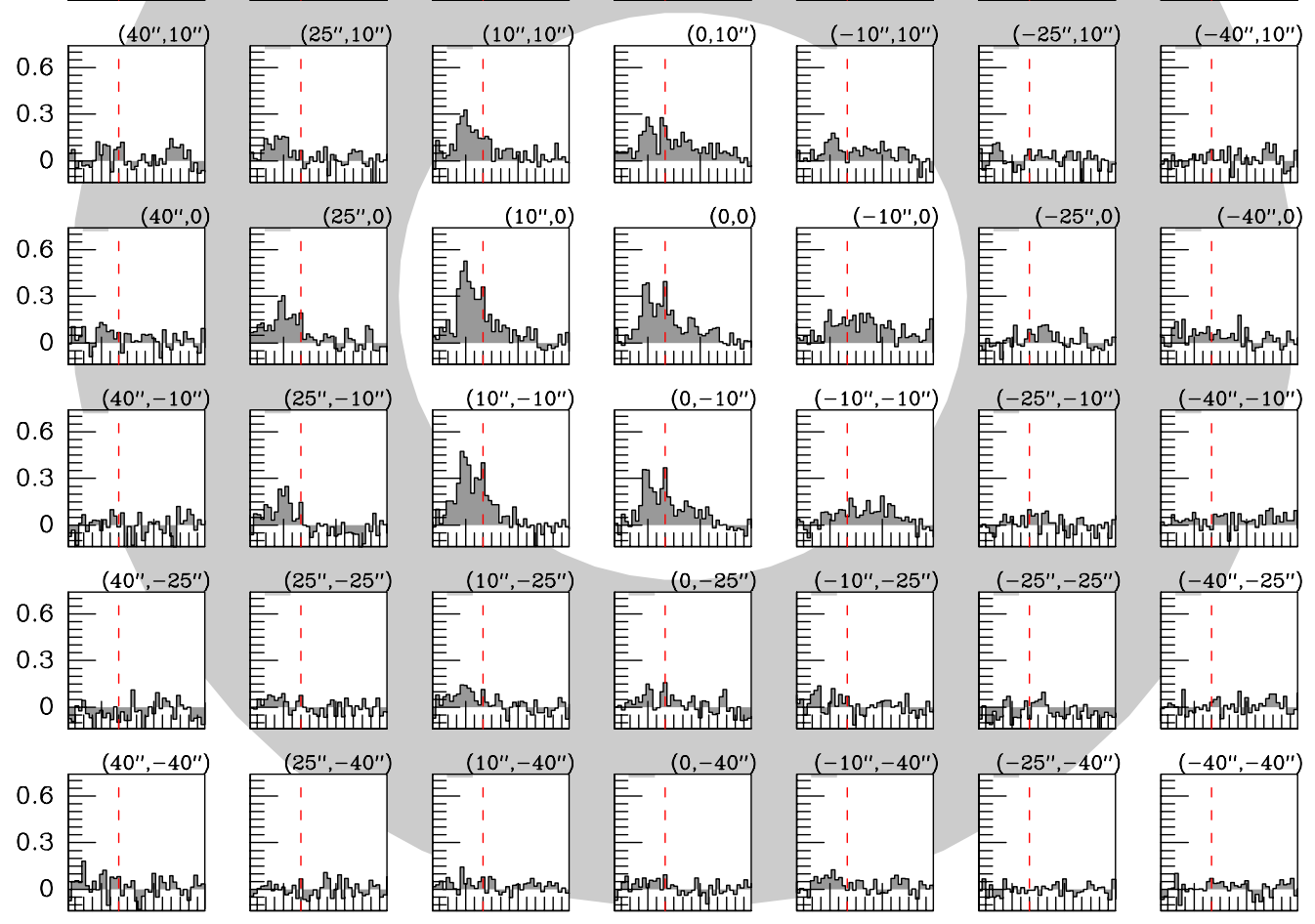

6090

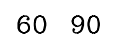

6090

6090

6090

Velocity (LSR, $\mathrm{km} / \mathrm{s})$

Fig. 4. Map of the APEX CO 2-1 emission (velocity resolution $2 \mathrm{~km} \mathrm{~s}^{-1}$ ) around IRAS 17163, with the star at $(0,0)$. The grey background ring shows the location and extent of the $25^{\prime \prime}$ dust ring. Most of the $\mathrm{CO}$ emission is found inside this dust ring. Note also the dashed red lines that mark $70 \mathrm{~km} \mathrm{~s}^{-1}$, dividing the blue and red components of the emission profile. These components are spatially separated, with the blue component peaking around $\left(10^{\prime \prime}, 0\right)$ and the red component around $\left(-10^{\prime \prime}, 0\right)$. The equivalent map for CO 3-2 is shown in Appendix A.

\section{Discussion}

\subsection{Velocity discrepancy}

IRAS 17163 shows broad CO emission, centred around $70 \mathrm{~km} \mathrm{~s}^{-1}$, with a velocity gradient across the circumstellar envelope. We hence take this to be the systemic velocity of the star. The star also shows multiple optical Fe II lines, centred on a velocity of $18 \mathrm{~km} \mathrm{~s}^{-1}$. This should also be indicative of the systemic velocity, yet it is blueshifted by $\sim 50 \mathrm{~km} \mathrm{~s}^{-1}$.

A velocity discrepancy between $\mathrm{CO}$ and optical line velocities is also found in the archetypal YHG IRC+10420, although it is smaller. In this object, the optical emission lines are blueshifted by $15-25 \mathrm{~km} \mathrm{~s}^{-1}$ compared with the CO systemic velocity at $+75 \mathrm{~km} \mathrm{~s}^{-1}$. However, the optical lines still fall within the CO velocity range. In the YHG HD 179821, the CO and optical lines coincide at $+100 \mathrm{~km} \mathrm{~s}^{-1}$.

There are several possible explanations for the large velocity discrepancy in IRAS 17163 . First is photospheric pulsations: yellow hypergiant stars are characterised by large-scale photospheric variability. The YHG $\rho$ Cas has shown radial velocity variations of up to $35 \mathrm{~km} \mathrm{~s}^{-1}$ associated with outburst activity, on a timescale of $\sim 100$ days (Lobel et al. 2003). Furthermore, models show that YHG pulsational variability can be up to $100 \mathrm{~km} \mathrm{~s}^{-1}$ (Fadeyev 2011). As we only have one epoch of optical observations of IRAS 17163, we cannot constrain its photospheric variability, so it is possible that our optical spectrum was taken near the peak of a pulsational cycle when the photospheric lines were blueshifted by $\sim 50 \mathrm{~km} \mathrm{~s}^{-1}$.

Another possibility is a close binary companion. In this case, the $\mathrm{CO}$ emission would indicate the systemic velocity of the binary system, while the Fe II lines arise only from our YHG. IRAS 17163 would need to have an orbital velocity of at least $50 \mathrm{~km} \mathrm{~s}^{-1}$ to explain the velocity discrepancy between the $\mathrm{CO}$ and Fe II lines. For example, the LBV binary MWC 314 has an orbital speed of $\sim 100 \mathrm{~km} \mathrm{~s}^{-1}$ (Lobel et al. 2013). The binary hypothesis also requires that the companion be relatively optically faint as there is no indication of lines at a very different velocity in the optical spectrum. The companion would have to be $\sim 100$ (the highest signal-to-noise ratio in the spectrum) times less luminous than IRAS 17163 , i.e., $\sim 5 \times 10^{3} L_{\odot}$. For a main sequence companion, this rules out only the heaviest (greater than $\sim 10 M_{\odot}$ ) stars. 


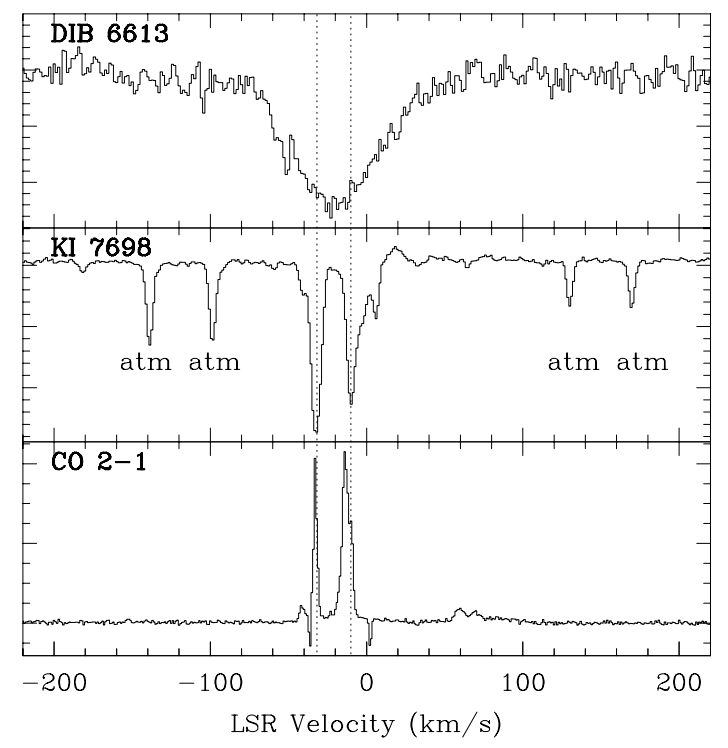

Fig. 5. Interstellar absorption lines from the optical spectrum, with wavelengths in $\AA$, at the same velocity as the interstellar CO emission from APEX observations. The dotted vertical lines are at -32 and $-10 \mathrm{~km} \mathrm{~s}^{-1}$.

In summary, the radial velocity of the Fe II optical lines is compatible with the systemic velocity derived from the $\mathrm{CO}$ observations. We note that a systemic velocity of $\sim 70 \mathrm{~km} \mathrm{~s}^{-1}$ means that the star has a very high peculiar velocity, redshifted by about $100 \mathrm{~km} \mathrm{~s}^{-1}$ compared with galactic rotation at $4 \mathrm{kpc}$ (Reid et al. 2009). However, about $20 \%$ of massive stars in the Milky Way are runaways (e.g. Mason et al. 1998), so this is not implausible.

\subsection{Nature of IRAS 17163}

\subsubsection{Distance}

The knowledge of the distance is a key parameter for defining the nature and physical properties of IRAS 17163, as it determines its absolute luminosity and hence its position in the HertzsprungRussell diagram. The signature of foreground interstellar clouds in its optical spectrum allowed Lagadec et al. (2011b) to conclude that the distance to IRAS 17163 is at least $3.6 \mathrm{kpc}$. This large distance is also supported by recent trigonometric parallax measurements of the star-forming region RCW 122, placing it at a distance of $3.38 \pm 0.3 \mathrm{kpc}$ (Wu et al. 2012). RCW 122 is part of the complex whose $\mathrm{CO}$ emission matches the $\mathrm{KI}$ absorption in our spectra (Fig. 5), and is hence in front of IRAS 17163.

The knowledge of the absolute magnitude, $M_{V}$ (see Sect. 3.2), of IRAS 17163 provides an alternative method of determining its distance, $D$ (in pc), using the equation

$m_{V}-M_{V}-A_{V}+5=5 \times \log D$,

where $m_{V}$ is the apparent visual magnitude and $A_{V}$ the visual extinction along the line of sight to the star. We take $m_{V}=$ $13.03 \mathrm{mag}$ and $E(B-V)=4.10 \mathrm{mag}$ from Le Bertre et al. (1989) and assume that there has been no significant variation in the star between the date of the photometric observations (1988) and the more recent spectroscopic observations of OI (2009), from which $M_{V} \sim-10$ is determined. A standard conversion between visual extinction and reddening of $A_{V}=3.1 \times E(B-V)$ is used. Taking the photometric reddening at face value, Eq. (1) gives a distance to IRAS 17163 of $\sim 1 \mathrm{kpc}$. Alternatively, an estimate of the interstellar reddening can be found from the depths

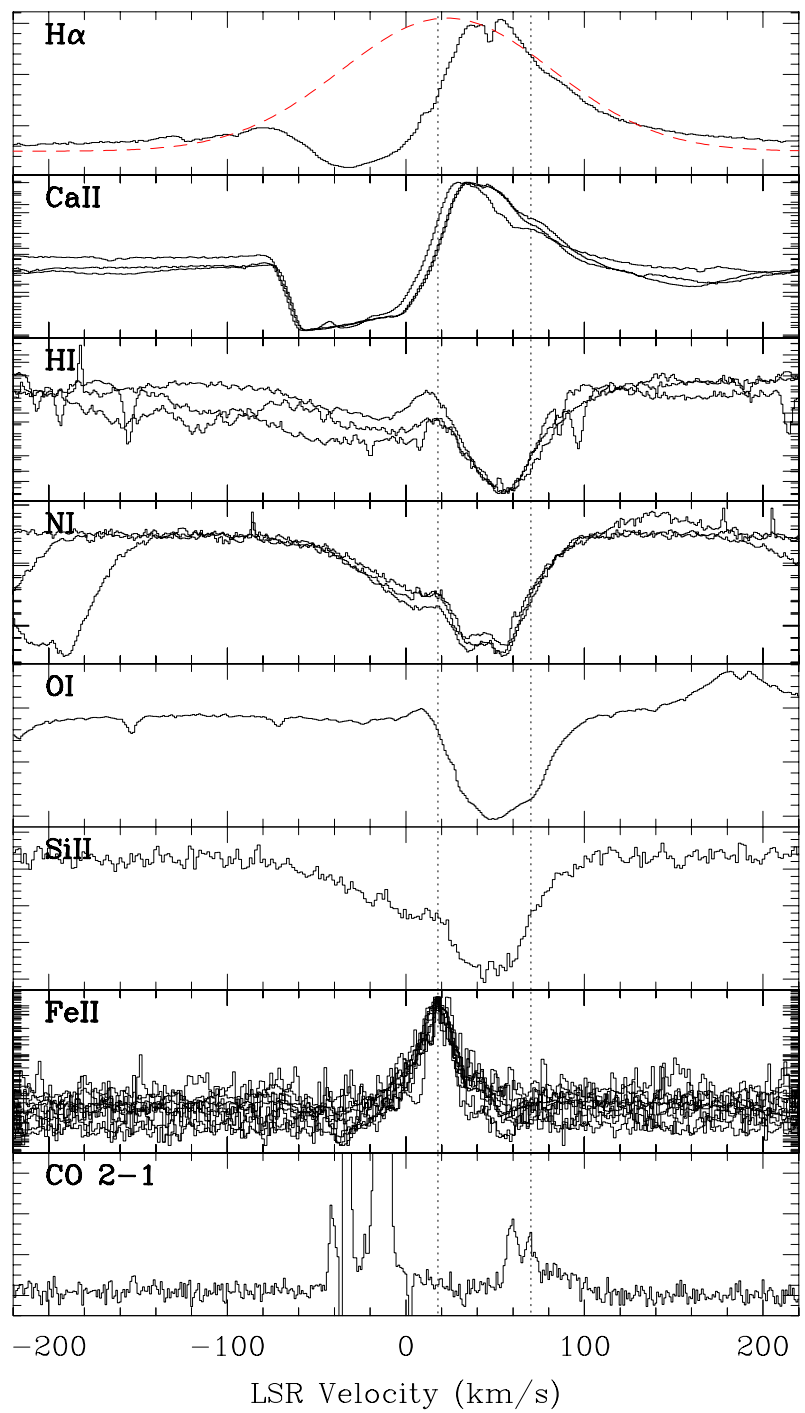

Fig. 6. Various lines from the optical spectrum. A Gaussian fit to the central part of the $\mathrm{H} \alpha$ line $\left(F W H M=140 \mathrm{~km} \mathrm{~s}^{-1}\right)$ is shown in dashed red. The dotted vertical lines are at 18 and $70 \mathrm{~km} \mathrm{~s}^{-1}$, matching the $\mathrm{Fe} \mathrm{II} \mathrm{and} \mathrm{centre} \mathrm{of} \mathrm{the} \mathrm{CO}$ emission, respectively. The $\mathrm{CO}$ emission at negative velocities is interstellar.

of DIBs in the optical spectrum. Taking the highest interstellar reddening value derived by Lagadec et al. (2011b), we calculate a distance of $\sim 7 \mathrm{kpc}$. The uncertainties in the amount and properties of interstellar and circumstellar dust limit the application of this method for constraining the distance to IRAS 17163. In any case, the distance interval $(\sim 1-7 \mathrm{kpc})$ is consistent with, but less restrictive than, that the 3.6-4.7 kpc found by Lagadec et al. (2011b). Throughout this paper we have assumed a distance of $4 \mathrm{kpc}$.

\subsubsection{Mass of the star}

The terminal velocities of stellar winds are typically of the order of the stellar escape velocity (see e.g. Abbott 1978; Lamers \& Cassinelli 1999), which we can use to roughly estimate the current stellar mass. We can estimate the wind terminal velocity from the Ca II P Cygni profile, where the blue edge of the absorption represents the highest wind speed: $-80 \mathrm{~km} \mathrm{~s}^{-1}$. As the wind originates at the systemic velocity, $70 \mathrm{~km} \mathrm{~s}^{-1}$, this gives a terminal velocity of $150 \mathrm{~km} \mathrm{~s}^{-1}$. 
From the luminosity of $5 \times 10^{5} L_{\odot}$ and an assumed effective temperature of $8000 \mathrm{~K}$ (from the determination of IRAS 17163 as a late B- or early A-type star by Lagadec et al. 2011b), we calculate a stellar radius of $\sim 370 R_{\odot}$. Then, equating the terminal velocity of the stellar wind, $150 \mathrm{~km} \mathrm{~s}^{-1}$, with the escape velocity $v_{\mathrm{e}}=\sqrt{2 G M_{*} / R_{*}}$, we derive a stellar mass of $\sim 22 M_{\odot}$. Summing this value with the estimate of the circumstellar mass (see Sect. 3.1) yields an initial mass of about $30 M_{\odot}$, consistent with the expected YHG initial mass range of 20 to $40 M_{\odot}$.

\subsubsection{Comparison with $\mathrm{IRC}+10420$}

There is a wealth of similarity between IRAS 17163 and IRC +10420 , the archetypal YHG. Their close proximity in the Hertzsprung-Russell diagram prompted the classification of IRAS 17163 as a YHG, and both objects have absolute magnitudes close to $M_{V}=-10$ (see Sect. 3.2). Both objects show distinct circumstellar shells, with ejection timescales of a few hundred years (Lagadec et al. 2011b; Oudmaijer et al. 2009).

Furthermore, the optical spectrum of IRAS 17163 closely resembles the peculiar optical spectrum of IRC +10420 (Oudmaijer 1998). The two spectra are shown, overlaid, in Appendix B. Both objects show strong $\mathrm{H} \alpha$ and $\mathrm{Ca}$ II lines. In IRAS 17163 these lines have clear P-Cygni profiles, indicative of circumstellar outflow. In IRC+10420 they show strong central absorptions. There are a few other differences between the two spectra: IRAS 17163 is somewhat more line rich, with about a dozen lines that are not seen at all in the spectrum of IRC +10420 . Conversely, there are two Fe I lines seen only in the IRC+10420 spectrum. The Mg II lines around $7890 \AA$ are seen in emission in IRAS 17163 and in absorption in IRC +10420.

Overall, the optical spectra of IRAS 17163 and IRC+10420 are very similar. This, along with the other similarities between these two objects, supports the classification of IRAS 17163 as a yellow hypergiant.

\section{Conclusions}

IRAS 17163 was recently proposed to be a member of the rare class of yellow hypergiants by Lagadec et al. (2011b) based on its location on a Hertzsprung-Russell diagram close to the archetypal yellow hypergiant IRC +10420 . To further investigate the nature of the star, we obtained $\mathrm{CO} J=2-1$ and $J=3-2$ APEX observations and a high-resolution Mercator spectrum between 5100 and $9000 \AA$ A. Our findings can be summarized as follows:

- We observe CO emission associated with IRAS 17163, at a radial velocity around $v_{\mathrm{LSR}}=+70 \mathrm{~km} \mathrm{~s}^{-1}$.

- The CO line profile is broad $\left(\Delta v \sim 60 \mathrm{~km} \mathrm{~s}^{-1}\right)$ and asymmetric, with a blue component showing multiple peaks and a fainter, broader red component.

- A velocity gradient is resolved across the circumstellar envelope: a $\sim 18^{\prime \prime}$ offset between the blue and red components' emission peaks, suggesting an asymmetrical outflow in an approximately east-west direction.
- There is a velocity discrepancy between the CO emission, at $\sim 70 \mathrm{~km} \mathrm{~s}^{-1}$, and the optical Fe II lines at $18 \mathrm{~km} \mathrm{~s}^{-1}$. This might be explained by large photospheric pulsations in the star or by a binary companion.

- The optical spectrum is line rich and very similar to the peculiar spectrum of the archetypal yellow hypergiant star IRC +10420 .

- The P-Cygni profiles of the $\mathrm{H} \alpha$ and Ca II lines signify a substantial amount of outflowing material.

- The absolute magnitude was estimated using the equivalent width of the O I (7774 $\AA$ ) triplet to be $M_{V}=-10$, comparable to that of IRC +10420 .

We conclude that the similarity in properties between IRAS 17163 and IRC+10420 reinforces the classification of IRAS 17163 as a yellow hypergiant. The $\mathrm{CO}$ emission from this object is complex and has raised questions about how it fits in with previous observations. Further observations of the circumstellar envelope at high angular resolution, for instance, with ALMA, will be required to reveal the distribution of the molecular gas and further study the mass-loss activity.

Acknowledgements. We would like to thank the director of the Onsala Space Observatory, Hans Olofsson, for generous allocation of DDT time. We also thank Per Bergman for his help with our APEX observations and for helpful discussions. This publication makes use of data products from the Widefield Infrared Survey Explorer, which is a joint project of the University of California, Los Angeles, and the Jet Propulsion Laboratory/California Institute of Technology, funded by the National Aeronautics and Space Administration.

\section{References}

Abbott, D. C. 1978, ApJ, 225, 893

Arellano Ferro, A., Giridhar, S., \& Rojo Arellano, E., et al. 2003, Rev. Mex. Astron. Astrophys., 39, 3

Arnal, E. M., Duronea, N. U., Testori, J. C. 2008, A\&A, 486, 807

Bergman, P., Parise, B., Liseau, R., et al. 2011, A\&A, 531, A8

Castro-Carrizo, A., Quintana-Lacaci, G., Bujarrabal, V., et al. 2007, A\&A, 465, 457

Dinh-V.-Trung, Muller, S., Lim, J., et al. 2009, ApJ, 697, 409

de Jager, C. 1998, A\&ARv, 8, 145

Fadeyev, Y. A. 2011, Astron. Lett., 37, 403

Henize, K. G. 1976, ApJS, 30, 491

Humphreys, R. M., Smith, N., Davidson, K., et al. 1997, AJ, 114, 2778

Humphreys, R. M., Davidson, K., \& Smith, N. 2002, AJ, 124, 1026

Hutsemékers, D., Cox, N. L. J., \& Vamvatira-Nakou, C. 2013, A\&A, 552, A6

Lagadec, E., Zijlstra, A. A., Oudmaijer, R. D., et al. 2011b, A\&A, 534, L10

Lamers, H. J. G. L. M., \& Cassinelli, J. P. 1999, Introduction to Stellar Winds (Cambridge University Press)

Le Bertre, T., Heydari-Malayeri, M., Epchtein, N., et al. 1989, A\&A, 225, 417

Lobel, A., Dupree, A. K., Stefanik R. P., et al. 2003, ApJ, 583, 923

Lobel, A., Groh, J. H., Martayan, C., et al. 2013, A\&A, 559, A16

Loup, C., Forveille, T., Omont, A., \& Paul, J. F. 1993, A\&AS, 99, 291

Mason, B. D., Gies, D. R., Hartkopf, W. I., et al. 1998, AJ, 115, 821

Oudmaijer, R. D., 1998, A\&AS, 129, 541

Oudmaijer, R. D., Groenewegen, M. A. T., Matthews, H. E., et al. 1996, MNRAS, 280, 1062

Oudmaijer, R. D., Davies, B., de Wit, W.-J., \& Patel, M. 2009, in The Biggest, Baddest, Coolest Stars, ASP Conf. Ser. 112, 17

Raskin, G., van Winckel, H., Hensberge, H., et al. 2011, A\&A, 526, A69

Reid, M. J., Menten, K. M., Zheng, X. W., et al. 2009, ApJ, 700, 137

Wu, Y. W., Xu, Y., Menten, K. M., et al. 2012, IAU Symp., 287, 425 


\section{Appendix A: Map of CO 3-2 emission}

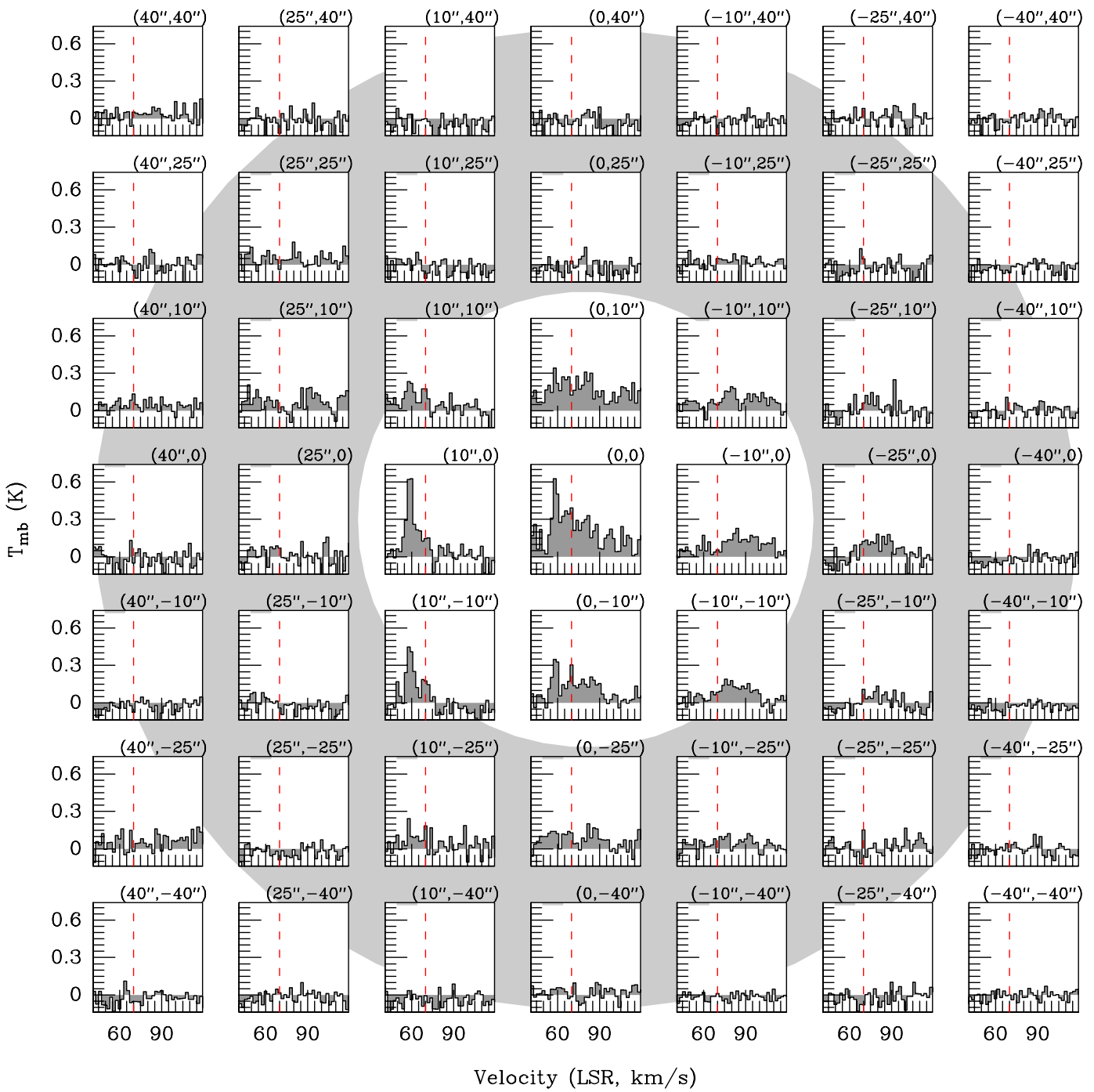

Fig. A.1. Map of the APEX CO 3-2 emission (velocity resolution $2 \mathrm{~km} \mathrm{~s}^{-1}$ ) around IRAS 17163 , with the star at (0, 0). The grey background ring shows the location and extent of the $25^{\prime \prime}$ dust ring. Most of the CO emission is found inside this dust ring. Note also the dashed red lines that mark $70 \mathrm{~km} \mathrm{~s}^{-1}$, dividing the blue and red components of the emission profile. These components are spatially separated, with the blue component peaking around $\left(10^{\prime \prime}, 0\right)$ and the red component around $\left(-10^{\prime \prime}, 0\right)$. 
Appendix B: Optical spectrum of IRAS 17163, overlaid with the spectrum of IRC+10420
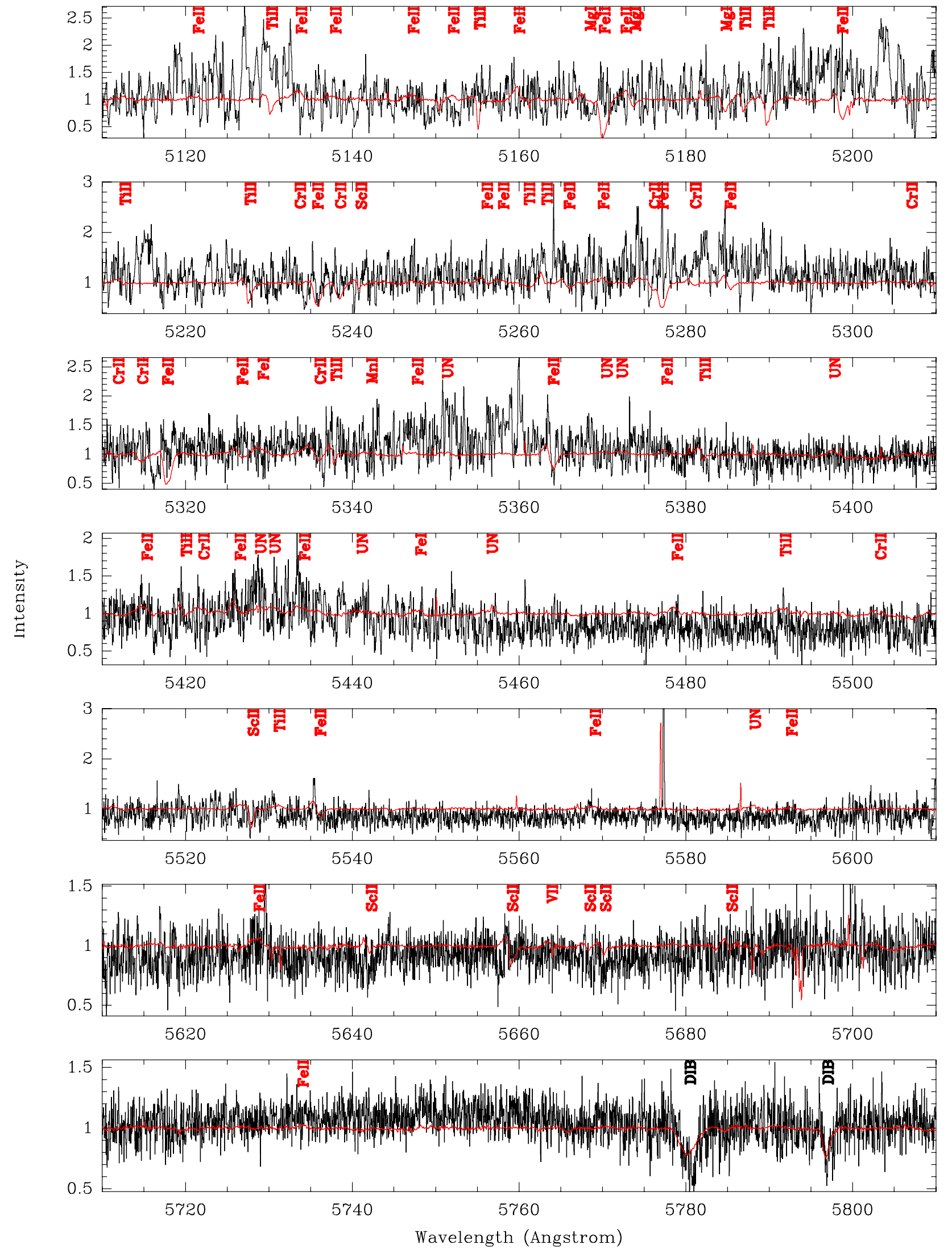

Fig. B.1. Optical spectrum of IRAS 17163, in black, overlaid with the spectrum of IRC+10420 (from Oudmaijer 1998), in red. The line identifications in red were made for the IRC+10420 spectrum by Oudmaijer (1998; with UN indicating an unidentified line, and H hydrogen recombination lines), and have been corrected for a systemic velocity of $75 \mathrm{~km} \mathrm{~s}^{-1}$. The Fe II identifications in black have been corrected for a line velocity of $18 \mathrm{~km} \mathrm{~s}^{-1}$. 
S. H. J. Wallström et al.: Investigating the nature of the Fried Egg nebula
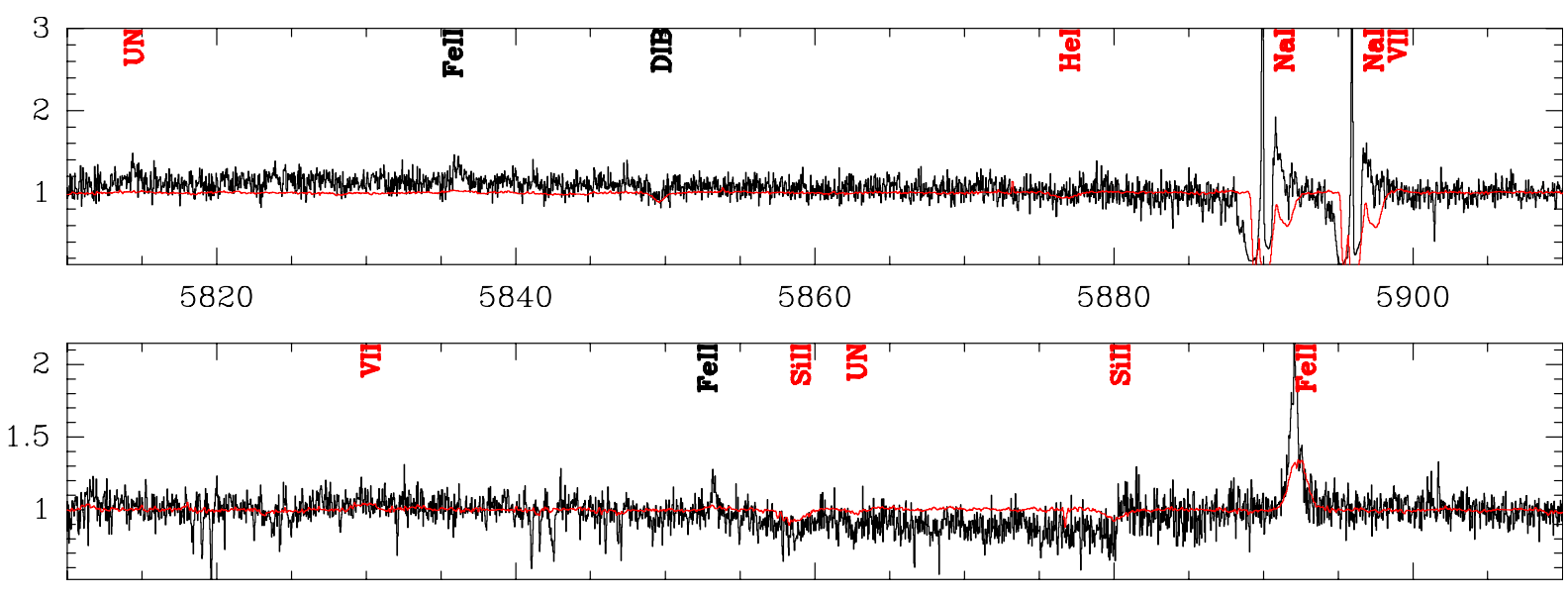
5920
5940
5960
5980
6000
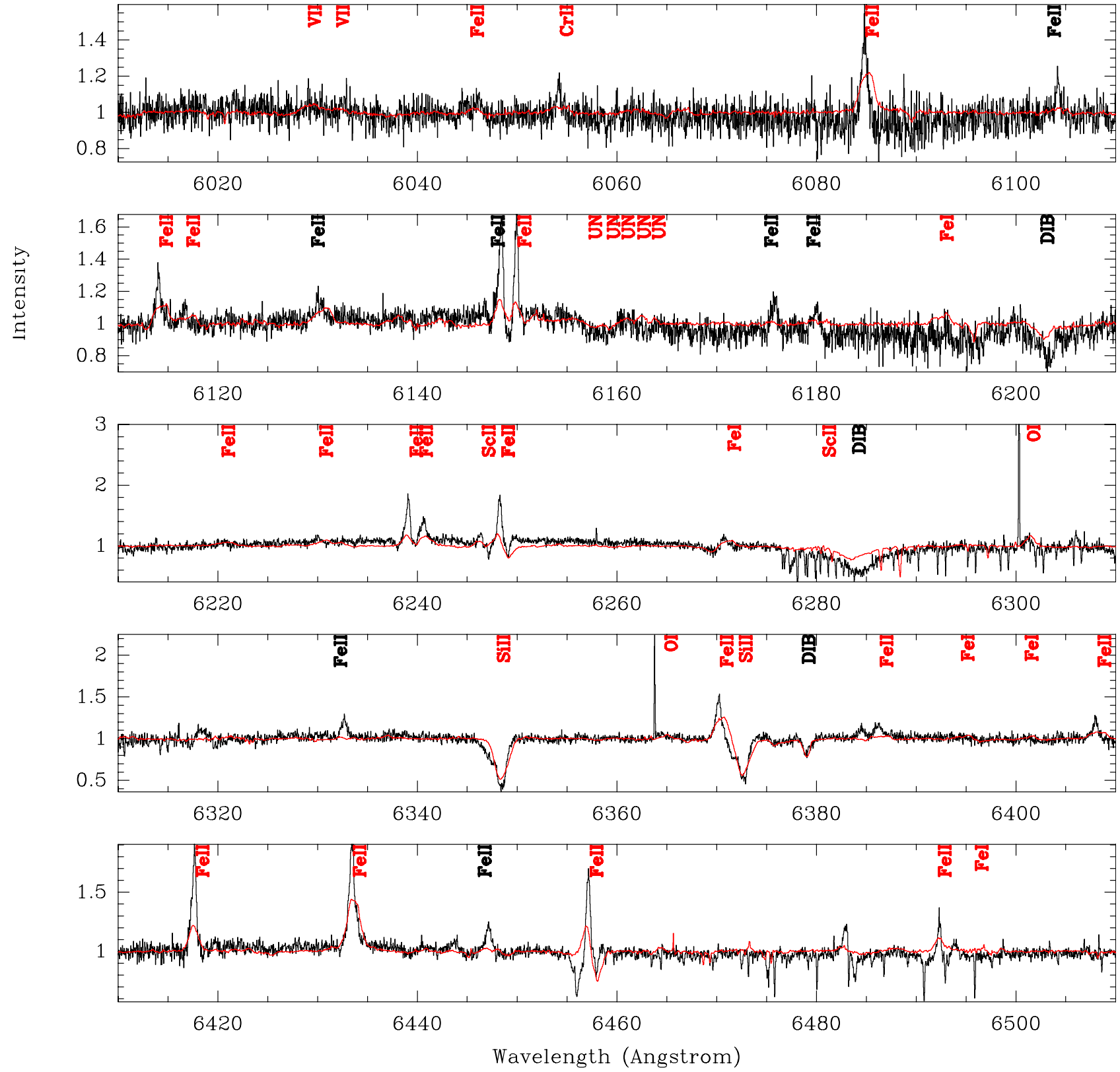

Fig. B.1. continued. 

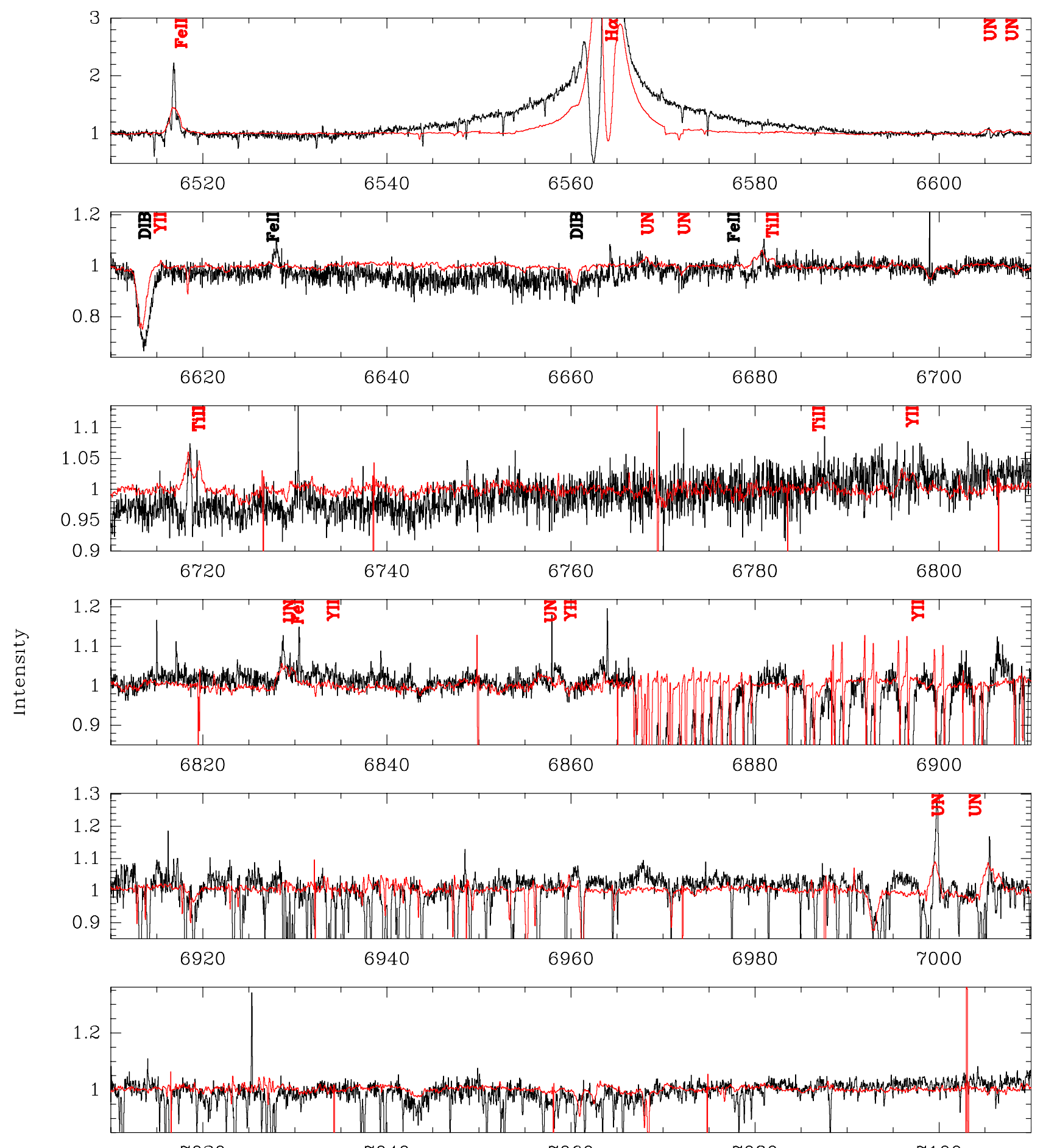

7020

7040

7060

7080

7100

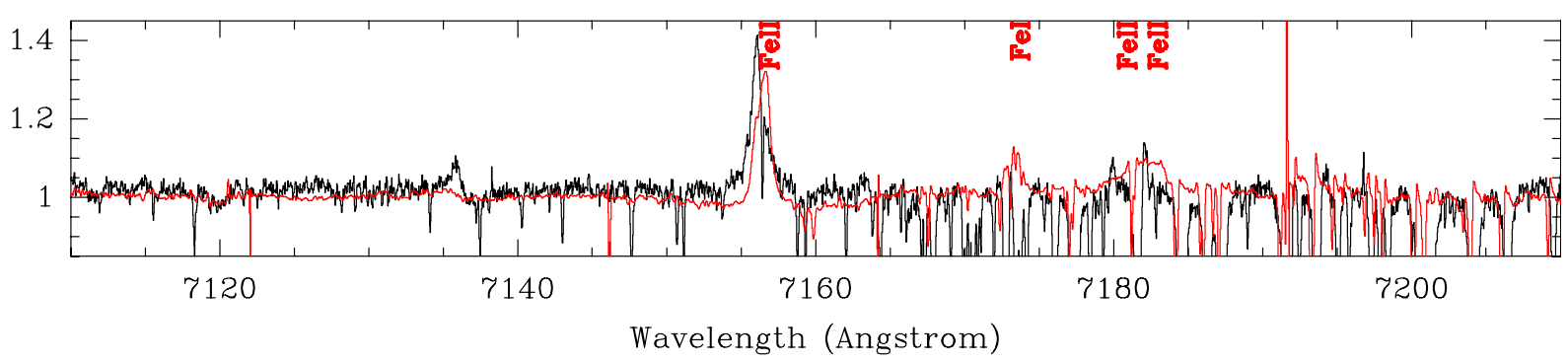

Fig. B.1. continued. 
S. H. J. Wallström et al.: Investigating the nature of the Fried Egg nebula
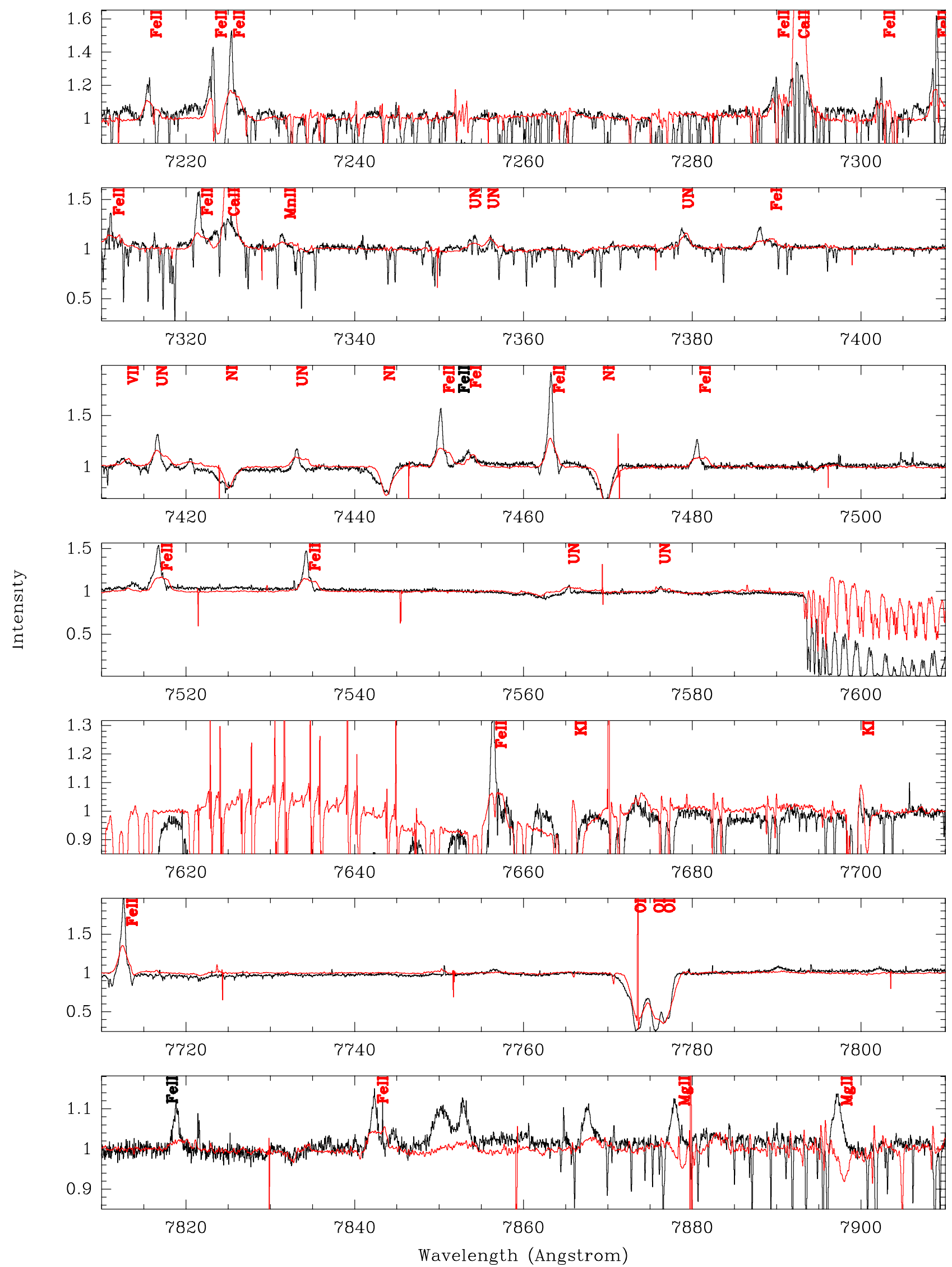

Fig. B.1. continued. 

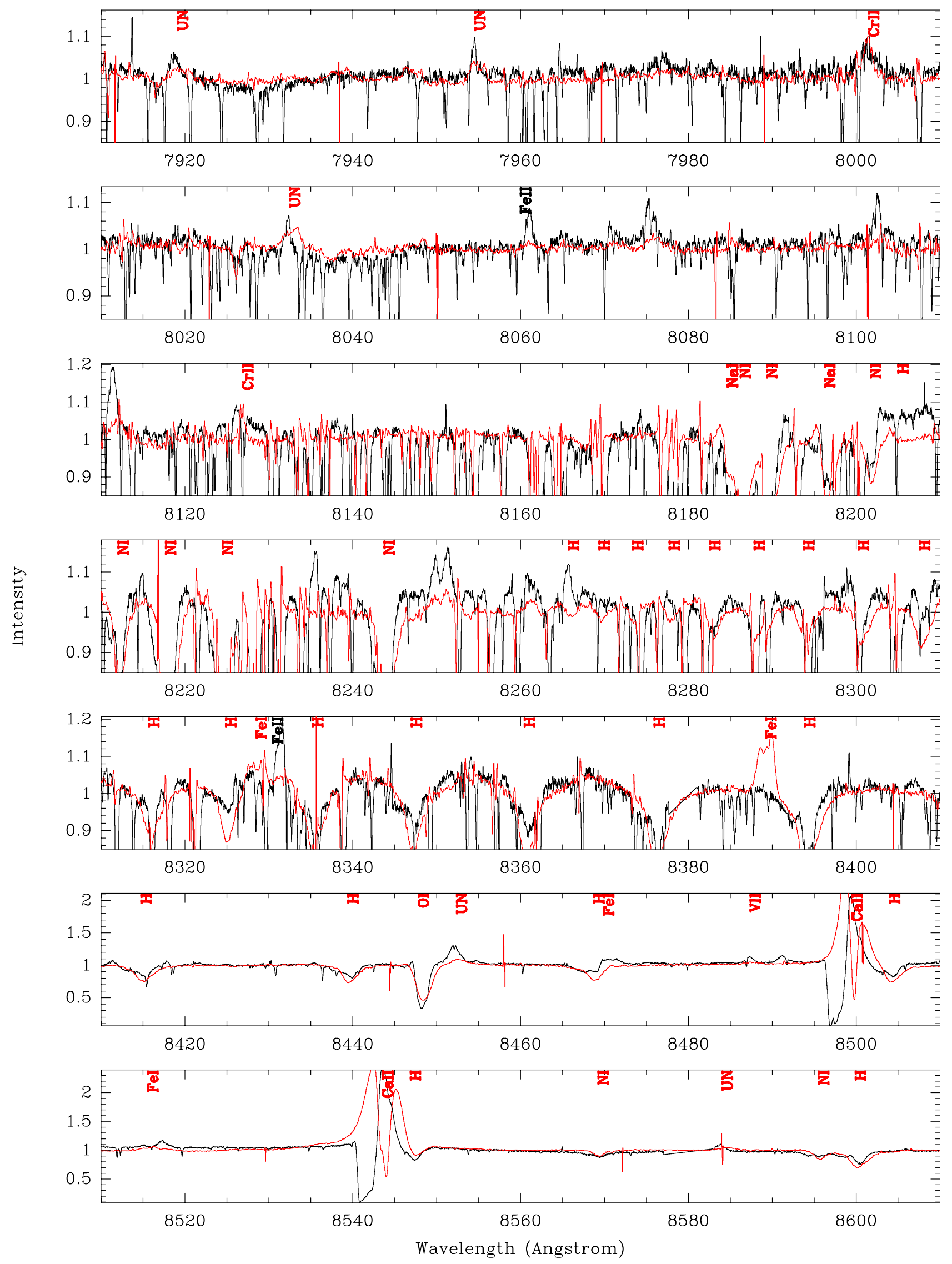

Fig. B.1. continued. 
S. H. J. Wallström et al.: Investigating the nature of the Fried Egg nebula
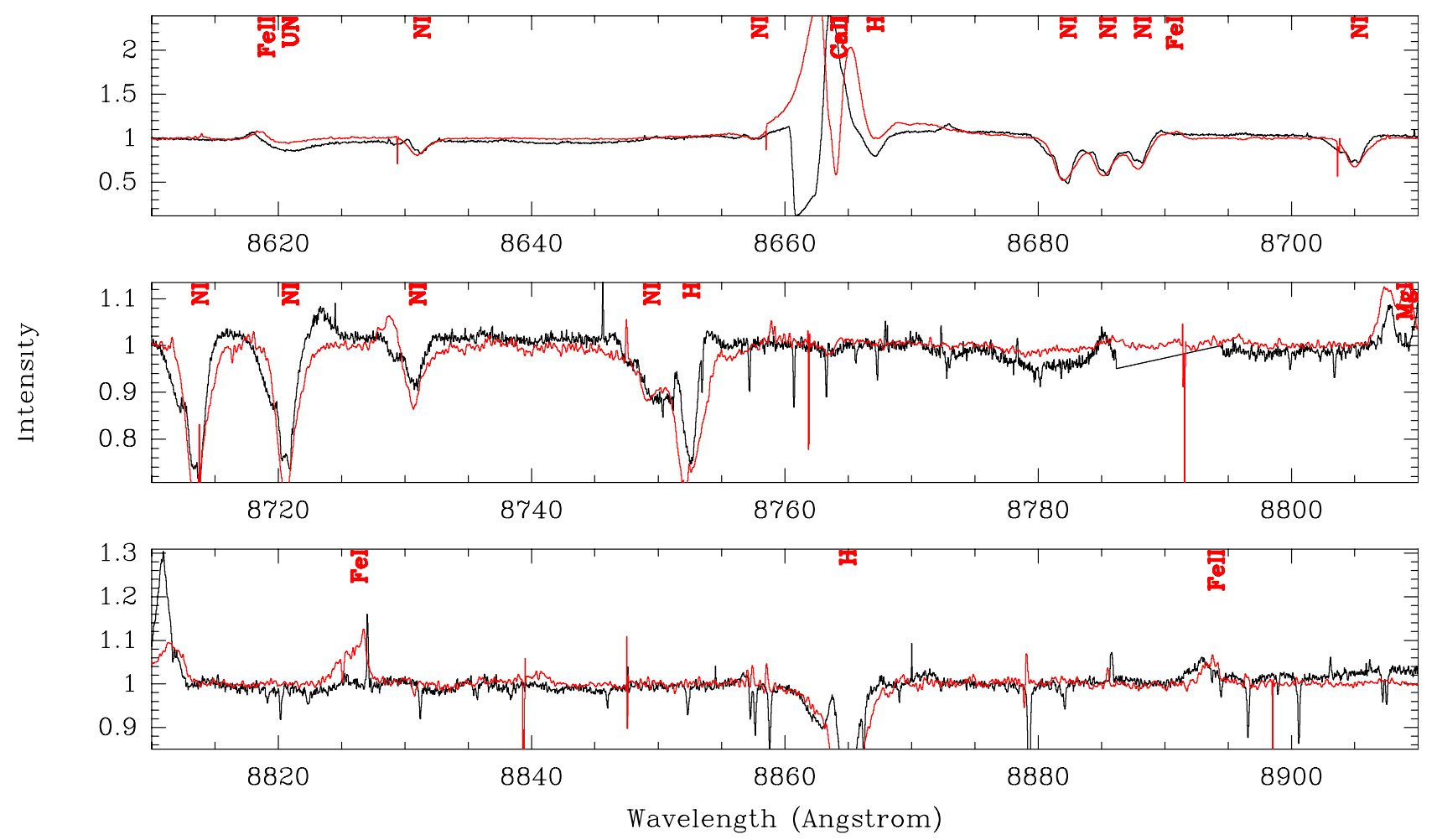

Fig. B.1. continued. 